\title{
Exploring the bulk of the BL Lac object population: parsec scale radio properties and gamma ray emission
}

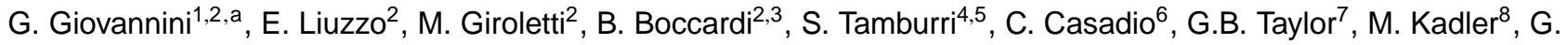 \\ Tosti $^{9}$, and A. Mignano ${ }^{2}$ \\ ${ }^{1}$ Dipartimento di Fisica e Astronomia, Universita' di Bologna, Italy \\ ${ }^{2}$ Istituto di Radioastronomia/INAF, Bologna, Italy \\ ${ }^{3}$ Max Planck Inst. für Radioastronomie, Bonn, Germany \\ ${ }^{4}$ Osservatorio Astronomico di Brera/INAF, Milano, Italy \\ ${ }^{5}$ Universita' dell'Insubria, Como, Italy \\ ${ }^{6}$ Inst. de Astrofisica de Andalucia/CSIC, Granada, Spain \\ ${ }^{7}$ Dept. of Physics and Astronomy, New Mexico University, USA \\ ${ }^{8}$ Universität Würzburg, Würzburg, Germany \\ ${ }^{9}$ Universita' di Perugia, Perugia, Italy
}

\begin{abstract}
BL Lac objects have been found to be the largest population of emitters in gamma-ray band. However, since they are relatively weak radio sources, their parsec scale structure and most of their radio properties are poorly known. To increase our knowledge of the BL Lac object population, we selected a sample of BL Lacs from the BZ Cat at low redshift $(\mathrm{z}<0.2)$, with no constrain on the radio flux density and gamma-ray activity. We present here the results of a first VLBA observation at 8 and $15 \mathrm{GHz}$ and shortly discuss their properties
\end{abstract}

\section{Introduction}

Based on results from the Energetic Gamma-Ray Experiment Telescope (EGRET) on the Compton Gamma-Ray Observatory (CGRO), it is well known that the main contribution to the $\gamma$-ray sky comes from the Galactic plane emission, pulsars, and blazars [11]. Among the latter class of objects, Flat Spectrum Radio Quasars (FSRQs) were the most numerous compared to the BL Lac objects, making up $77 \%$ of the high confidence blazar associations. With the advent of the Fermi mission, studies of a large number of $\gamma$-ray sources have become possible thanks to the unprecedented sensitivity of the Large Area Telescope (LAT, [5]). In contrast with the previous EGRET results, the LAT has shown that the BL Lacs, and not the FSRQs, are now the most common $\gamma$-ray emitters: in the Clean 2 LAC Sample, 395 are BL Lac objects, 310 FSRQs, 157 sources of unknown type, 22 other AGNs, and 2 starburst galaxies [3], [4].

Big questions are nevertheless remaining open, such as the $\gamma$-ray origin and location, the distance of the main energy dissipation site from the nucleus, and the relation between the $\gamma$-ray and radio emission.

BL Lac objects belong to the blazar population showing a high core dominance, large degrees of variability and polarization, and one-sided Doppler beamed parsec-scale jets. However, they present properties quite different from

\footnotetext{
a e-mail: ggiovann@ira.inaf.it
}

those of FRSQs: as e.g. their lower apparent velocities (see e.g. [7], [14], [13]).

Thanks to Fermi findings, the high energy characteristics of BL Lacs can be investigated, but to understand the origin and the nuclear properties of these sources more information at different frequencies are necessary. Very Long Baseline Interferometer (VLBI) campaigns are one of the most incisive observational tools to address the questions opened by the Fermi results.

From a detailed literature review, it is evident that previous VLBI surveys have looked at the parcsec scale properties of the brightest BL Lac objects, while the majority of the BL Lac population is below their flux density limits [19], [9], [10], [18], [6]. In fact, many LAT BL Lacs are highfrequency-synchrotron-peaked (HSP) sources, discovered at X-rays and generally they are faint radio sources, seldom studied with VLBI. To gain a deeper understanding of BL Lac properties, we selected a sample of low redshift BL Lac sources with no selection limits on their radio flux density or high frequency emission. In this work, we present our analysis of this sample of nearby BL Lacs. We observed all sources at 8 and $15 \mathrm{GHz}$ with the VLBA for about $1 \mathrm{hr}$. Targets weaker than $50 \mathrm{mJy}$ in NVSS have been observed in phase reference mode at 15 $\mathrm{GHz}$, sources weaker than $30 \mathrm{mJy}$ have been observed in phase reference mode at 8 and $15 \mathrm{GHz}$.

The intrinsic parameters quoted in this paper are computed with $\Lambda$ CDM cosmology with $H_{0}=71 \mathrm{~km} \mathrm{~s}^{-1} \mathrm{Mpc}^{-1}$, $\Omega_{m}=0.27$, and $\Omega_{\Lambda}=0.73$. 


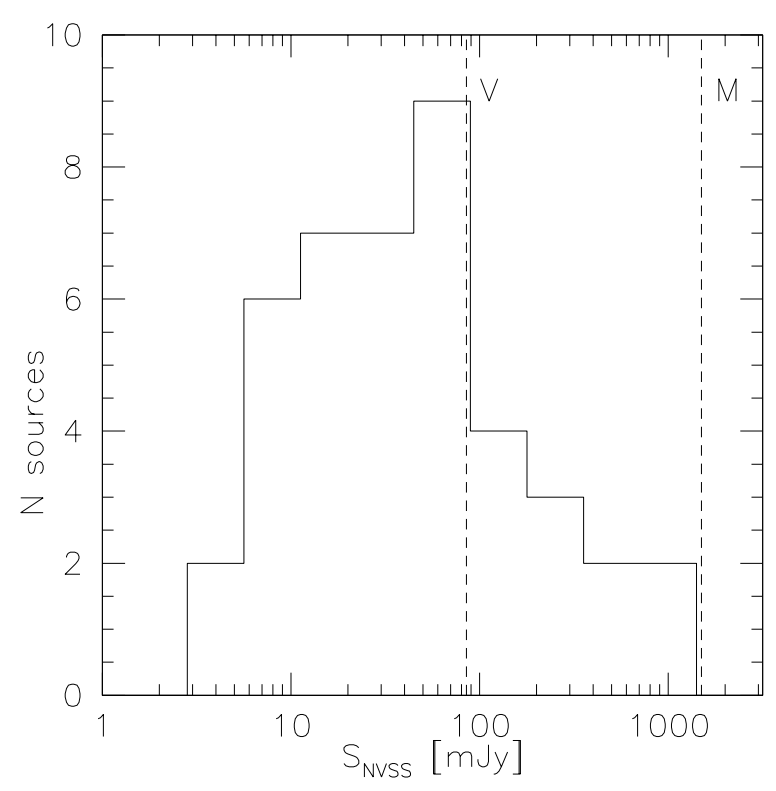

Figure 1. Distribution of the NVSS flux density for sources in our sample. The Dashed lines show the flux density limits of the MOJAVE-1 and VIPS surveys (see text).

\section{The sample}

We selected our sample of BL Lacs from the Roma BZCat Catalog of known blazars [17] with two constraints:

- a measured redshift $\mathrm{z}<0.2$,

- BL Lacs located within the sky area covered by the Sloan Digital Sky Survey (SDSS, [1]).

Our two selection criteria on the BZ Cat allow us

1) to investigate also the least powerful sources, such as the weak population of HSP BL Lacs, with a good linear resolution;

2) a good characterization not only of the optical properties, but also of their extended radio characteristics as the Faint Images of the Radio Sky at Twenty-Centimeters (FIRST) cover the same SDSS field.

The total number of BL Lacs in our sample is 42 (see [16] for more details). The National Radio Astronomy Observatory (NRAO) VLA Sky Survey Catalog (NVSS) flux density distribution for the sources in the sample is shown in Fig. 1. The flux limits of the Monitoring of jets in active galactic nuclei (AGN) with Very Long Baseline Array (VLBA) Experiments (MOJAVE-1) (correlated flux $\mathrm{S}>$ $1.5 \mathrm{Jy}$ at $2 \mathrm{~cm},[14])$ and VLBA Imaging and Polarimetry Survey (VIPS) ( $>85 \mathrm{mJy}$ at $5 \mathrm{GHz},[12])$ surveys are also plotted (assuming $\alpha=0.0$ ). Fig. 1 points out that our sample is representative of a population which is the majority of the whole BL Lacs but it is unexplored by two of the most complete previous VLBI surveys.

\section{Results}

\subsection{Detection rate}

In our sample, 27/42 (64\%) objects show parsec-scale radio emission. In particular, 24 BL Lac objects are revealed at both frequencies, while two are detected only at $8 \mathrm{GHz}$ $(\mathrm{J} 1221+0821$ and $\mathrm{J} 1231+6414)$ and one only at $15 \mathrm{GHz}$ $(\mathrm{J} 1427+5409)$ [16]. We note that many of the sources revealed using phase referencing show a flux density below the noise level of sources observed without this technique. This suggests that more sources could be detected using the phase referencing mode for all the observations (e.g. J1427+5409). Point-like morphologies are present in $8 / 27(31 \%)$ of the detected sources at $8 \mathrm{GHz}$, and in $14 / 26$ $(54 \%)$ of the revealed targets at $15 \mathrm{GHz}$. The other detected sources show an one-sided structure. We note that in our sample, among the 8 BL Lacs having one-sided morphology at both frequencies, 7 are the most luminous (and bright) BL Lacs at mas scale.

\subsection{Radio structures}

Most of our sources show a parsec-scale flux density significantly lower than expected from the kiloparsec-scale flux densities estimated from the NVSS and/or FIRST radio surveys. In most cases, this difference cannot be due to the radio spectrum or to variability, and it suggests the presence of a sub-kiloparsec radio structure lost in VLBA data because of sensitivity and the lack of short baselines. To better understand this point, we evaluated, for all objects, the source compactness (SC) value defined as the ratio of the $8 \mathrm{GHz}$ VLBA and the NVSS total powers. Moreover, we estimated the core dominance (CD) of each BL Lac of the sample as defined in [15]. The core dominance is the ratio between the observed core radio power and the expected core radio power estimated from the unbeamed total radio power at low frequency, according to the relation given in [8]. The core dominance is a strong indication of the Doppler factor for each source and it allows for us to estimate constraints for the jet velocity and orientation. Since in most of our sample a measure of the total radio power at low frequency is unavailable,we estimated the total radio power at $408 \mathrm{MHz}$ extrapolating the NVSS total radio power using an average spectral index $=$ 0.7 . For the core radio power we used the radio power at 8 $\mathrm{GHz}$.

We show the SC in Fig. 2 and the CD distributions for the present sample in Fig. 3. We added also the CD of radio sources (radio galaxies and 2 BL Lacs) from the Bologna Complete Sample (BCS, see [15]) for a comparison. As expected, the distribution of BL Lac sources peaks at higher core dominance according to unified models. Looking at the derived values of source compactness and core dominance, together with the source morphology and radio spectrum, the BL Lac objects of our sample could be divided as follows:

- Doppler Dominated (DD) sources: Among the 42 BL Lacs, we have 23 sources in which the emission is dominated by the central core. They are characterized by a core dominance always larger than 5.5 suggesting that they are relativistic Doppler boosted objects. We note that a core dominance larger than 5.5 implies a jet velocity $0.8 \mathrm{c}$ or faster and a jet orientation with respect to the line of sight smaller than $30^{\circ}$. Most of these sources display 


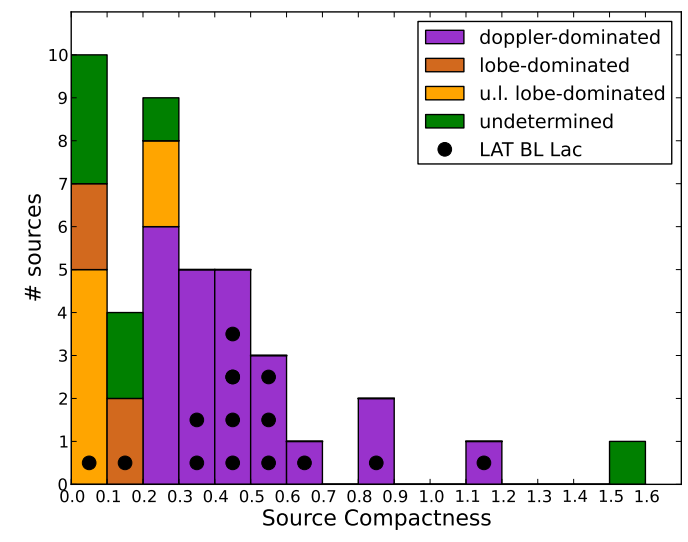

Figure 2. Source Compactness (SC as defined in Sect. 3.2) distribution. Upper limits are shown in yellow (u.1.).

a core dominant structure and a one-sided jet, confirming the importance of relativistic effects. The SC is generally high, even if in some case the missing flux is important, suggesting the presence of a structure more complex than the detected one-sided jet. For comparison, we note that radio galaxies of the BCS [15] show in most cases a core dominance smaller than 4 and in many cases smaller than 1 (Fig. 3).

- Lobe Dominated (LB) sources: In 11 cases, the VLBI total fluxes are a small fraction of the kiloparsecscale ones, with core dominance $<5.5$. This group includes both undetected and detected sources in VLBA maps. In a few sources, the radio spectrum is quite steep, suggesting that they are dominated by an extended steep spectrum structure despite their classification as BL Lac objects.

- Undetermined (U) objects: There are 8 sources that are not detected in our VLBA images. Their arcsec flux density is low, therefore we cannot say that the lack of detection is because no compact core is present or it is a sensitivity problem. Deeper observations will be necessary to investigate their parsec-scale properties.

\section{Gamma-ray properties}

We searched for gamma-ray counterparts of our BL Lac sample: 14/42 show high energy emission in the Second LAT AGN catalog (2LAC, [3], [4]). We call them LAT BL Lacs.

Among these LAT BL Lacs, 2 (J0847+1133 and $\mathrm{J} 1534+3715)$ are classified as LD objects, the others are DD objects. Moreover, the LD BL Lac J1534+3715 is the only LAT sources not detected by our VLBA observations. We note that LAT BL Lac objects have higher on average values of the core dominance with respect to the non LAT ones (Fig. 3), confirming the idea that these sources are dominated by Doppler boosting effects (note that the two BL Lacs of the BCS sample: Mrk 421 and Mrk 501, are LAT sources and are in agreement with the properties of LAT BL Lacs in our sample).

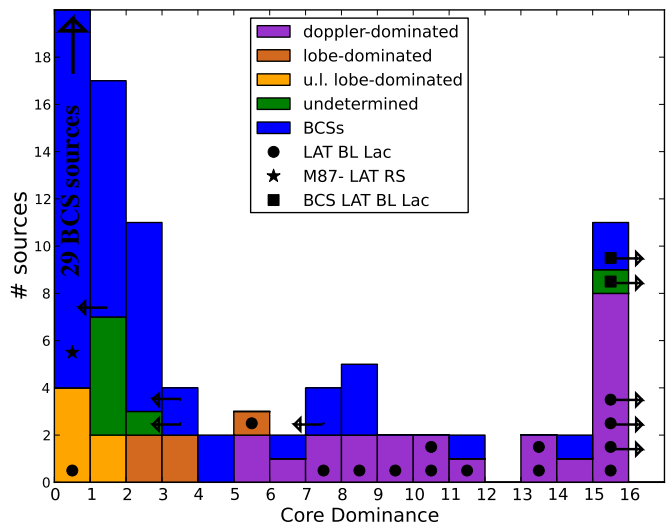

Figure 3. Core Dominance (CD see Sect. 3.2) distribution. Arrows indicate upper and lower limit $\mathrm{CD}$ values.

In Fig. 4, we plot the $8 \mathrm{GHz}-\mathrm{VLBA}$ radio flux density versus the gamma-ray fluxes: sources show a good correlation with the exception of $\mathrm{J} 1419+5423$, which has a very high radio flux density $\left(\mathrm{S}_{V L B A, 8 G H z} \sim 969.5 \mathrm{mJy}\right)$, but a modest $\gamma$-ray flux. This source also has $\mathrm{SC}=1.19$, and very high core dominance, with the VLBA flux density greater than the kiloparsec-scale flux density. Therefore, it is likely that it is a variable source observed with VLBA during an outburst.

We estimated the Pearson index correlation $r$ for these data and we found $r=0.90$, which means that a linear correlation between $\log S_{V L B A, 8 G H z}$ and $\log S_{\gamma}$ is present. We performed a linear fit $\left(\log \mathrm{S}_{\gamma}=a \log \mathrm{S}_{V L B A, 8 G H z}+b\right)$ finding $a=0.69$ and $b=-9.97$ (see dashed line in Fig. 4). The correlation is in agreement with results of [2] for BL Lac objects.

\section{Discussion and Conclusions}

We selected a sample of nearby BL Lacs with no constraint on their radio or high energy flux density. Our aim is to investigate their nuclear properties given that one of the surprising results of Fermi mission is that BL Lac objects, and not FSRQs, are the most common gamma-ray emitters in the sky. VLBA images for our entire sample have been presented in [16]. From VLBI observations, we found that most of the BL Lacs in our sample (23/42) show a high Core Dominance ( $>5.5$ ), in agreement with expectations from unified models. We classified these sources as Doppler Dominated objects. They show a strong evidence of an active nuclear region affected by Doppler boosting effects, confirmed by the Fermi detection of 12 of these sources, and by the correlation between the radio flux density and gamma-ray flux. However, in many sources $(14 / 23)$ the $\mathrm{SC}$ is lower than 0.5 , suggesting that in many BL Lac sources a significant sub-kiloparsec structure is present. Of the remaining sources, 11/42 were classified as Lobe Dominated sources. In these BL Lacs, the core dominance is lower than 5.5 and there is no evidence of a relativistic nuclear structure or strong boosing effects. 


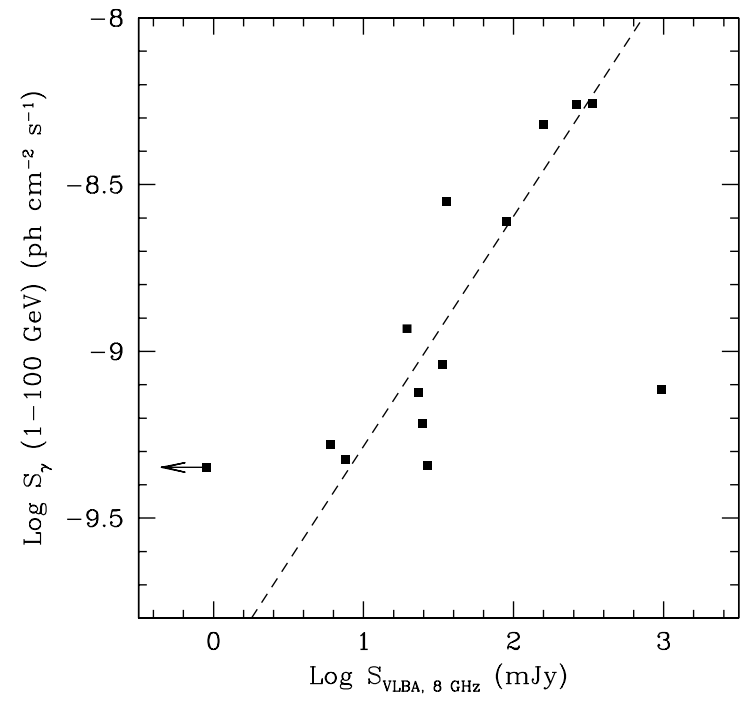

Figure 4. $\log \mathrm{S}_{V L B A, 8 G H z}$ vs $\log \mathrm{S}_{\gamma}$. for the 14 LAT BL Lacs in our sample. The arrow indicates the VLBA $8 \mathrm{GHz}$ flux density upper limit for $\mathrm{J} 1534+3715$, not detected in our data. The point at the right side, outside the correlation, refers to J1419+5432, see text.

This fraction is higher than expected in a sample of BL Lac sources. Among these objects, 2 have been detected by Fermi: J0847+1133 and J1534+3715. In the case of $\mathrm{J} 0847+1133$, we have a VLBI detection, and the core dominance is 5.1 , therefore we can consider this source as a source with intermediate properties. J1534+3715 was not detected in our VLBA observations and we suggest that it could be a misaligned object. Its core dominance is lower than 0.8. We note that, among the radio galaxies of the BCS sample [15], only M87 shows gamma-ray emission and its core dominance is 0.9 . We were unable to classify the other 8 objects in our sample because of their low flux densities. From a comparison of the distribution of the core dominance obtained for our BL Lac sample and for the radio galaxies of the BCS sample [15], we can suggest the presence of two populations: beamed (core dominance $\sim 5.5$ or higher) and un-beamed objects (core dominance lower than $\sim 5.5$ ). We suggest that BL Lacs classified as LD objects could be intermediate or misclassified sources, but more multi-wavelength data are necessary to investigate this point. The origin of gamma-ray emission in sources in the two classes could be different: strongly related to the relativistic jets in DD, but not in LD.
More data are necessary to better investigate this scenario and the radio structure of $\mathrm{J} 1534+3715$ needs to be confirmed. In some DD sources (6/23) and all LD objects, the $\mathrm{SC}$ is lower than 0.3 , suggesting the presence of a relevant sub-kiloparsec structure. The morphology and the properties of this extended radio emitting region will be analyzed with future observations that could help in understanding the difference between DD and LD BL Lacs and the connection with the high energy emission.

\section{References}

[1] Abazajian, K.N., Adelman-McCarthy, J.K., Agüeros, M.A., et al., ApJS 182, 543 (2009)

[2] Abdo, A.A., Ackermann, M., Ajello, M., et al., ApJ, 715, 429 (2010)

[3] Ackermann, M., Ajello, M., Allafort, A., et al. ApJ 741, 30 (2011)

[4] Ackermann, M., Ajello, M., Allafort, A., et al. ApJ 743, 171 (2011)

[5] Atwood, W.B., Abdo, A.A., Ackermann, M., et al. ApJ 697, 1071 (2009)

[6] Cassaro, P., Stanghellini, C., Dallacasa, d., et al., A\&A 381, 378 (2002)

[7] Gabuzda, D.C., Mullan, C.M., Cawthorne, T.V., et al. ApJ 435, 140 (1994)

[8] Giovannini, G., Feretti, L., Venturi, T., et al., ApJ, 435, 116 (1994)

[9] Giroletti, M., Giovannini, G., Taylor, G.B., Falomo, R., Apj 613, 752 (2004)

[10] Giroletti, M., Giovannini, G., Taylor, G.B., Falomo, R. ApJ 646, 801 (2006)

[11] Hartman, R.C.,Bertsch, D.L., Bloom, S.D., et al. ApJS 123, 79 (1999)

[12] Helmboldt, J.F., Taylor, G.B., Tremblay,S., et al., ApJ, 658, 203 (2007)

[13] Lico, R., Giroletti, M., Orienti, M., et al. A\&A 545, A117 (2012)

[14] Lister, M.L., Cohen, M.H., Homan, D.C., et al., AJ 138, 1874 (2009)

[15] Liuzzo, E., Giovannini, G., Giroletti, M., Taylor, G.B. A\&A, 505, 509 (2009)

[16] Liuzzo, E., Giroletti, M., Giovannini, G., etr al., A\&A in press, arXiv:1309.2774 (2013)

[17] Massaro, E., Giommi, P., Leto, C., et al. A\&A, 495, 6 (2009)

[18] Rector T.A., Gabuzda, D.C., Stcke, J.T., AJ 125, 1060 (2003)

[19] Wu, Z., Jiang, D.R., Gu, M., Liu, Y. A\&A 466, 63 (2007) 